Pacific

Journal of

Mathematics

\title{
THE BERGMAN KERNEL FOR CIRCULAR MULTIPLY CONNECTED DOMAINS
}

Moonja JeOng ANd Vladimir MityusheV

Volume $233 \quad$ No. 1

November 2007 


\title{
THE BERGMAN KERNEL FOR CIRCULAR MULTIPLY CONNECTED DOMAINS
}

\author{
Moonja JeOng And Vladimir MityusheV
}

\begin{abstract}
The Bergman kernel is written in terms of a Poincaré series for every circular multiply connected domain in the plane. The proposed exact formula is based on the Green's function constructed by Mityushev and S. V. Rogosin for multiply connected domains. First we verify the relation between the Green's function and the Bergman kernel for unbounded domains. Then we apply it to construct the Bergman kernel.
\end{abstract}

\section{Introduction}

The Bergman kernel plays an important role in studies of the boundary behavior of biholomorphic mappings, and of the potential theory in one complex variable. It is related to the Green's function and to other domain functions such as the Neumann function, harmonic measures, canonical mapping functions, and extremal problems [Bergman 1950; Bell 1992; Aharonov et al. 2005]. Therefore, explicit expressions of the Bergman kernels yield profound progress in the theory of functions.

The Bergman kernel represents the Bergman projection, which reproduces all holomorphic square integrable functions. In the unit disc $U$ in $\mathbb{C}$ with area measure $d x \wedge d y=i / 2 d z \wedge d \bar{z}$, the Bergman kernel is given by

$$
K_{U}(z, \zeta)=\frac{1}{\pi} \frac{1}{(1-z \bar{\zeta})^{2}}, \quad z, \zeta \in U
$$

The Bergman kernel $K_{\Omega}(z, \zeta)$ associated with a simply connected planar domain $\Omega$ is expressed by the Riemann mapping $f: \Omega \rightarrow U$

$$
K_{\Omega}(z, \zeta)=\frac{1}{\pi} \frac{f^{\prime}(z) \overline{f^{\prime}(\zeta)}}{(1-f(z) \overline{f(\zeta}))^{2}} \quad z, \zeta \in \Omega
$$

MSC2000: primary 30C20, 30C40; secondary 20C35, 30E25.

Keywords: Bergman kernel, Green's function, harmonic measure, multiply connected domain.

Jeong was supported by the Korea Research Foundation Grant R04-2003-000-10045-0 funded by the Korean Government. 
Conversely, the derivative of $f(z)$ is determined through the Bergman kernel by the formula

$$
f^{\prime}(z)=K_{\Omega}(z, \zeta) \sqrt{\frac{\pi}{K_{\Omega}(\zeta, \zeta)}},
$$

where the point $\zeta$ is transformed onto zero by the mapping $f$ with $f^{\prime}(\zeta)>0$.

The Bergman kernel for a circular annulus $A_{\rho}=\{z \in \mathbb{C}: \rho<|z|<1\}$ was calculated in [Bergman 1950, p. 10]

$$
K_{A_{\rho}}(z, \zeta)=\frac{1}{\pi z \bar{\zeta}}\left(\mathscr{P}(\ln z \bar{\zeta})+\frac{\eta_{1}}{\pi i}-\frac{1}{2 \ln \rho}\right),
$$

where $\mathscr{P}$ is the Weierstrass function with the periods $\omega_{1}=\pi i, \omega_{2}=\ln \rho$, and $\eta_{1}$ is the half-increment of the Weierstrass $\zeta$-function related to the period $\omega_{1}$. Ball and Clancey [1996] gave a formula for the Bergman kernel for multiply connected domains in terms of theta functions defined on the Jacobian variety of the Schottky double of domain. However, this formula involves the principal functionals of the Schottky double of domain for which only theorems of existence are known.

Note that in the several dimensional complex space, the Bergman kernel was calculated explicitly for the following domains: the unit ball, the polydisc, the Thullen domain [D'Angelo 1994], convex domains [Boas et al. 1999], the Lie ball [Hua 1963], the minimal ball [Oeljeklaus et al. 1997], and so on. For example, the Bergman kernel associated with the Thullen domain $\left\{\left(z_{1}, z_{2}\right) \in \mathbb{C}^{2}:\left|z_{1}\right|^{2 p}+\right.$ $\left.\left|z_{2}\right|^{2}<1\right\}$, with any positive integer $p$ is

$$
\sum_{k=1}^{2} c_{k} \frac{\left(1-z_{2} \bar{\zeta}_{2}\right)^{-2+(k / p)}}{\left(\left(1-z_{2} \bar{\zeta}_{2}\right)^{1 / p}-z_{1} \bar{\zeta}_{1}\right)^{k+1}}
$$

where $c_{1}=(p-1) /\left(\pi^{2} p\right)$ and $c_{2}=2 /\left(\pi^{2} p\right)$.

Let $M$ be a 1-dimensional complex manifold and $H^{2}(M)$ the Hilbert space of holomorphic 1-forms $h$ on $M$ such that

$$
\frac{i}{2} \int_{M} h \wedge \bar{h}<\infty .
$$

Let $h_{1}, h_{2}, \cdots$ be an orthonormal basis for $H^{2}(M)$. The Bergman kernel $\mathscr{K}_{M}$ associated with $M$ can be defined as follows:

$$
\mathscr{K}_{M}=\frac{i}{2} \sum_{k=1}^{\infty} h_{k} \wedge \bar{h}_{k} .
$$

In this paper, we consider domains with $C^{\infty}$ smooth boundary. The relation between the Bergman kernel and the Green's function is given by the following proposition: 
Proposition 1.1 [Schiffer 1946; Bell 1992, p. 131]. Suppose $\Omega$ is a bounded domain with $C^{\infty}$ smooth boundary. The Green's function $G_{\Omega}(z, w)$ and the Bergman kernel $K_{\Omega}(z, w)$ associated with $\Omega$ are related via

$$
K_{\Omega}(z, \zeta)=-\frac{2}{\pi} \frac{\partial^{2} G_{\Omega}(z, \zeta)}{\partial z \partial \bar{\zeta}}
$$

In this paper we write the Bergman kernel explicitly for every circular multiply connected domain. Our work is based on the Green function constructed in [Mityushev and Rogosin 2000] for multiply connected domains. In Section 2 we verify the relation (1-7) for unbounded domains. In Section 3 we apply it to the construction of the Bergman kernel.

\section{Green's function and the Bergman kernel}

On the first page of his book, B. Epstein [Epstein 1965] writes "Let $D$ denote any bounded* domain...", and in the footnote: "The restriction of boundedness is made for the sake of simplicity; all arguments to be presented in the present chapter and in the following chapters are valid, with at most minor modifications, for an unbounded domain whose boundary contains at least one component consisting of more than a single point." Since the present paper directly concerns such unbounded domains, we include in this section the formal derivation of the statements that we will need, as they seem not to be explicitly available elsewhere.

First, the definition of a biholomorphic mapping for unbounded domains is given. Let $\overline{\mathbb{C}}$ denote the extended complex plane $\mathbb{C} \cup\{\infty\}$. Following [Shabat 1985] we define a subset $U$ of $\overline{\mathbb{C}}$ to be open if

(1) $U \cap \mathbb{C}$ is open in $\mathbb{C}$, and

(2) if $\infty \in U$, then for some $R>0$, the set $\{z \in \mathbb{C}:|z|>R\}$ is contained in $U$.

Hence, the mapping of $\overline{\mathbb{C}}$ to $\overline{\mathbb{C}}$ given by $z \mapsto 1 / z$ is continuous. With the topology in $\overline{\mathbb{C}}$ and with the associated idea of continuity, we may think of meromorphic functions as holomorphic functions with values in $\overline{\mathbb{C}}$. According to [Greene and Krantz 2002, p. 143], we define a continuous function $F: U \rightarrow \overline{\mathbb{C}}$ on an open subset $U$ of $\overline{\mathbb{C}}$ to be holomorphic if

(1) $\left.F\right|_{F^{-1}(\mathbb{C})}$ is holomorphic and

(2) $F^{-1}(\{\infty\})$ is a discrete set in $U$, and $1 / F$ is holomorphic on some open neighborhood of $F^{-1}(\{\infty\})$.

Let $\Omega$ be a bounded domain with $C^{\infty}$ smooth boundary. For a fixed point $\zeta \in \Omega$, the Green's function $G_{\Omega}(z, \zeta)$ associated with $\Omega$ is defined by

$$
G_{\Omega}(z, \zeta)=-\ln |z-\zeta|+g_{\zeta}(z)
$$


where $g_{\zeta}(z)$ is the harmonic function of $z$ on $\Omega$ which solves the Dirichlet problem

$$
\left.g_{\zeta}(z)\right|_{\partial \Omega}=\ln |z-\zeta|
$$

Now we consider unbounded circular multiply connected domains. Introduce mutually disjoint discs

$$
D_{k}=\left\{z \in \mathbb{C}:\left|z-a_{k}\right|<r_{k}\right\}, \quad k=1,2, \ldots, n,
$$

and the complement of these discs to the extended complex plane

$$
D=\overline{\mathbb{C}}-\bigcup_{k=1}^{n}\left(D_{k} \cup \partial D_{k}\right)
$$

The domain $D$ is called a circular multiply connected domain.

The transformation formula for the Bergman kernels holds under biholomorphic mappings between two bounded domains. It is proved by a change of variable produced by a biholomorphic mapping between two bounded domains; see [Greene and Krantz 2002, p. 434]. The same arguments can be applied to a biholomorphic mapping between an unbounded multiply connected domain and a bounded multiply connected domain. For convenience, we present this result in the following lemma and sketch the proof below.

Lemma 2.1. Suppose that $f: D \rightarrow \Omega$ is a biholomorphic mapping where $D$ is defined by (2-1)-(2-2) and $\Omega$ is a bounded domain. The Bergman kernels $K_{D}$ and $K_{\Omega}$ associated with $D$ and $\Omega$ respectively, are related via

$$
K_{D}(z, \zeta)=f^{\prime}(z) K_{\Omega}(f(z), f(\zeta)) \overline{f^{\prime}(\zeta)} \quad z, \zeta \in D
$$

Proof. Introduce the function $\tilde{K}(z, \zeta)=f^{\prime}(z) K_{\Omega}(f(z), f(\zeta)) \overline{f^{\prime}(\zeta)}$ for $z, \zeta \in D$. Then

$$
\int_{D}|\tilde{K}(z, \zeta)|^{2} d A_{z}=\int_{D}\left|f^{\prime}(z)\right|^{2}\left|K_{\Omega}(f(z), f(\zeta))\right|^{2}\left|f^{\prime}(\zeta)\right|^{2} d A_{z} .
$$

By a change of variable, this becomes

$$
\begin{aligned}
\int_{\Omega}\left|f^{\prime}\left(f^{-1}(w)\right)\right|^{2}\left|K_{\Omega}(w, f(\zeta))\right|^{2}\left|f^{\prime}(\zeta)\right|^{2}\left|\left(f^{-1}\right)^{\prime}(w)\right|^{2} d A_{w} & \\
& =\left|f^{\prime}(\zeta)\right|^{2} \int_{\Omega}\left|K_{\Omega}(w, f(\zeta))\right|^{2} d A_{w}<\infty
\end{aligned}
$$

Now for any holomorphic function $\varphi \in L^{2}(D)$,

$$
\int_{D} \tilde{K}(z, \zeta) \varphi(\zeta) d A_{\zeta}=\int_{D} f^{\prime}(z) K_{\Omega}(f(z), f(\zeta)) \overline{f^{\prime}(\zeta)} \varphi(\zeta) d A_{\zeta}
$$




$$
\begin{aligned}
& =\int_{\Omega} f^{\prime}(z) K_{\Omega}(f(z), \eta) \overline{f^{\prime}\left(f^{-1}(\eta)\right)} \varphi\left(f^{-1}(\eta)\right)\left|\left(f^{-1}\right)^{\prime}(\eta)\right|^{2} d A_{\eta} \\
& =f^{\prime}(z) \int_{\Omega} K_{\Omega}(f(z), \eta)\left\{\left(\varphi \circ f^{-1}\right)(\eta)\left(f^{-1}\right)^{\prime}(\eta)\right\} d A_{\eta} \\
& =f^{\prime}(z)\left\{\left(\varphi \circ f^{-1}\right)(f(z))\left(f^{-1}\right)^{\prime}(f(z))\right\},
\end{aligned}
$$

since $\left(\varphi \circ f^{-1}\right)(\eta)\left(f^{-1}\right)^{\prime}(\eta)$ is holomorphic in $\Omega$ and belongs to $L^{2}(\Omega)$. Hence,

$$
\int_{D} \tilde{K}(z, \zeta) \varphi(\zeta) d A_{\zeta}=\varphi(z)
$$

By the uniqueness of the Bergman kernel, we establish that (2-3) is valid.

More generally, Lemma 2.1 can be applied to a biholomorphic mapping between two complex manifolds with the same dimensions. Suppose that $f: M_{1} \rightarrow M_{2}$ is a biholomorphic mapping where $M_{1}$ and $M_{2}$ are 1-dimensional complex manifolds. The Bergman kernels $\mathscr{K}_{M_{1}}$ and $\mathscr{K}_{M_{2}}$ associated with $M_{1}$ and $M_{2}$ respectively, are related via

$$
\mathscr{K}_{M_{1}}=(f \times f)^{*} \mathscr{K}_{M_{2}},
$$

where $f \times f$ is a biholomorphic mapping from $M_{1} \times M_{1}$ to $M_{2} \times M_{2}$.

Example 2.2. We apply (2-3) to get the Bergman kernel associated with the upper half plane $\mathbb{C}^{+}$. Let $f: \mathbb{C}^{+} \rightarrow U$ be the bilinear transformation

$$
f(z)=\frac{A\left(z-z_{0}\right)}{z-\bar{z}_{0}}
$$

where $\operatorname{Im} z_{0}>0$ and $|A|=1$. Then $f^{\prime}(z)=A\left(z_{0}-\bar{z}_{0}\right) /\left(z-\bar{z}_{0}\right)^{2}$ and

$$
K_{U}(f(z), f(\zeta))=\frac{1}{\pi(1-f(z) \overline{f(\zeta)})^{2}}=\frac{\left(z-\bar{z}_{0}\right)^{2}\left(\bar{\zeta}-z_{0}\right)^{2}}{\pi(\bar{\zeta}-z)^{2}\left(z_{0}-\bar{z}_{0}\right)^{2}}
$$

Therefore

$$
K_{\mathbb{C}^{+}}(z, \zeta)=f^{\prime}(z) K_{U}(f(z), f(\zeta)) \overline{f^{\prime}(\zeta)}=-\frac{1}{\pi(z-\bar{\zeta})^{2}}
$$

The Bergman kernel function (2-9) does vanish at $z=\infty$, while the Bergman kernel form $-d z d \bar{\zeta} /\left(\pi(z-\bar{\zeta})^{2}\right)$ is nonvanishing on the closure of $\mathbb{C}^{+} \times \mathbb{C}^{+}$.

The Green's function is invariant under biholomorphic mappings between two bounded domains with $C^{\infty}$ smooth boundaries; see [Conway 1978, p. 276; Bell 1992, p. 128]. We extend this result to the case of biholomorphic mappings between $D$ and bounded domains with $C^{\infty}$ smooth boundaries. 
Lemma 2.3. Let the domain $D$ be defined by (2-1)-(2-2) and $\Omega$ be a bounded domain in the complex plane with $C^{\infty}$ smooth boundary. If $f$ is a biholomorphic mapping of $D$ onto $\Omega$, then the Green's functions $G_{D}$ and $G_{\Omega}$ associated with $D$ and $\Omega$ respectively, satisfy the identity

$$
G_{D}(z, \zeta)=G_{\Omega}(f(z), f(\zeta)), \quad z, \zeta \in D .
$$

Proof. It is enough to show that $G_{\Omega}(f(z), f(\zeta))$ is the Green's function for $D$ with singularity at $z=\zeta \in D$. Note that $f \in C^{\infty}(\bar{D})$ since $D$ has $C^{\infty}$ smooth boundary and $\bar{D}$ is compact. We can write $f(z)-f(\zeta)=(z-\zeta) H_{\zeta}(z)$ where $H_{\zeta}(z)$ is a nonvanishing holomorphic function in $A^{\infty}(D)=C^{\infty}(\bar{D}) \cup \mathscr{H}(D)$. Here $\mathscr{H}(D)$ represents the set of holomorphic functions on $D$. Hence,

$$
\ln |f(z)-f(\zeta)|=\ln |z-\zeta|+\ln \left|H_{\zeta}(z)\right|
$$

where $\ln \left|H_{\zeta}(z)\right|$ is a harmonic function in $C^{\infty}(\bar{D})$. Hence $G_{\Omega}(f(z), f(\zeta))+$ $\ln |z-\zeta|$ is a harmonic function of $z$ on $D$ with

$$
G_{\Omega}(f(z), f(\zeta))_{\mid z \in \partial D}=0 .
$$

Therefore, by the uniqueness of Green's functions, $G_{\Omega}(f(z), f(\zeta))$ is the Green's function associated with $D$; see [Conway 1978, p. 276].

Using Lemma 2.3 we obtain the following result.

Theorem 2.4. Let the domain $D$ be defined by (2-1)-(2-2). The Bergman kernel $K_{D}$ and the Green's function $G_{D}(z, \zeta)$ associated with $D$ are related via

$$
K_{D}(z, \zeta)=-\frac{2}{\pi} \frac{\partial^{2} G_{D}(z, \zeta)}{\partial z \partial \bar{\zeta}}
$$

Proof. Let $f: D \rightarrow \Omega$ be a biholomorphic mapping of $D$ onto a bounded domain $\Omega$ with $C^{\infty}$ smooth boundary. By Proposition 1.1,

$$
K_{\Omega}(w, \eta)=-\frac{2}{\pi} \frac{\partial^{2} G_{\Omega}(w, \eta)}{\partial w \partial \bar{\eta}}
$$

for $w, \eta \in \Omega$ where $K_{\Omega}(w, \eta)$ is the Bergman kernel and $G_{\Omega}(w, \eta)$ is the Green's function associated with $\Omega$. Application of Lemma 2.1 and (2-12) yields

$$
K_{D}(z, \zeta)=f^{\prime}(z) K_{\Omega}(w, \eta) \overline{f^{\prime}(\zeta)}=f^{\prime}(z)\left(-\frac{2}{\pi} \frac{\partial^{2} G_{\Omega}(w, \eta)}{\partial w \partial \bar{\eta}}\right) \overline{f^{\prime}(\zeta)}
$$

where $w=f(z), \eta=f(\zeta)$. By a change of variables, this becomes

$$
-\frac{2}{\pi} f^{\prime}(z) \frac{\partial^{2} G_{D}(z, \zeta)}{\partial z \partial \bar{\zeta}}\left(f^{-1}\right)^{\prime}(w) \overline{\left(f^{-1}\right)^{\prime}(\eta)} \overline{f^{\prime}(\zeta)}=-\frac{2}{\pi} \frac{\partial^{2} G_{D}(z, \zeta)}{\partial z \partial \bar{\zeta}} .
$$




\section{Exact formula for the Bergman kernel}

Let $D$ be a circular multiply connected domain described by (2-1)-(2-2). Hereafter we deal only with the domain $D$, hence for brevity we omit the corresponding indexes in the Green and Bergman functions.

Schottky group. Consider inversions with respect to the circles $\left|z-a_{k}\right|=r_{k}$ and their compositions

$$
z_{(k)}^{*}:=\frac{r_{k}^{2}}{\overline{z-a_{k}}}+a_{k}, z_{\left(k_{s} k_{s-1} \ldots k_{1}\right)}^{*}:=\left(z_{\left(k_{s-1} \ldots k_{1}\right)}^{*}\right)_{\left(k_{s}\right)}^{*} .
$$

In the sequence $k_{1}, k_{2}, \ldots, k_{s}$ no two neighboring numbers are equal. When $s$ is even, these are Möbius transformations in $z$. If $s$ is odd, we have transformations in $\bar{z}$. Hence, the mappings can be written in the form

$$
\begin{array}{ll}
\gamma_{j}(z)=\left(e_{j} z+b_{j}\right) /\left(c_{j} z+d_{j}\right), & s \in 2 \mathbb{Z}, \\
\gamma_{j}(\bar{z})=\left(e_{j} \bar{z}+b_{j}\right) /\left(c_{j} \bar{z}+d_{j}\right), & s \in 2 \mathbb{Z}+1,
\end{array}
$$

where $e_{j} d_{j}-b_{j} c_{j}=1$. Here $\gamma_{0}(z):=z$ (identity mapping), $\gamma_{1}(\bar{z}):=z_{(1)}^{*}, \ldots$, $\gamma_{n}(\bar{z}):=z_{(n)}^{*}\left(n\right.$ simple inversions), $\gamma_{n+1}(z):=z_{(12)}^{*}, \gamma_{n+2}(z):=z_{(13)}^{*}, \ldots, \gamma_{n^{2}}(z):=$ $z_{(n, n-1)}^{*}\left(n^{2}-n\right.$ pairs of inversions $), \gamma_{n^{2}+1}(\bar{z}):=z_{(121)}^{*}, \ldots$ and so on. The number $s$ is called the level of the mapping $\gamma_{j}$. The indexes $j$ of $\gamma_{j}$ are ordered in such a way that the level is not decreasing. The functions (3-1) or (3-2) generate a Schottky group $\mathscr{Y}$. Thus, each element of $\mathscr{Y}$ is presented in the form of the composition of inversions (3-1) or in the form of linearly ordered functions (3-2). Let $\mathscr{T}_{m}$ be the subset of $\mathscr{Y}-\left\{\gamma_{0}\right\}$ consisting of mappings whose last inversion is different from $z_{(m)}^{*}$, i.e., $\mathscr{S}_{m}=\left\{z_{\left(k_{s} k_{s-1} \ldots k_{1}\right)}^{*}: k_{s} \neq m\right\}$.

Harmonic measures. For a fixed point $z_{0} \in D-\{\infty\}$, we introduce the functions

$$
\psi_{m}(z)=\ln \left(\prod_{\gamma_{j} \in \mathscr{Y}_{m}} \psi_{m}^{(j)}(z)\right),
$$

where we have, for even level of $\gamma_{j} \in \mathscr{S}$

$$
\psi_{m}^{(j)}(z)=\frac{\gamma_{j}(z)-a_{m}}{\gamma_{j}\left(z_{0}\right)-a_{m}},
$$

and for odd level of $\gamma_{j} \in \mathscr{Y}$

$$
\psi_{m}^{(j)}(z)=\frac{\overline{\gamma_{j}\left(\bar{z}_{0}\right)-a_{m}}}{\overline{\gamma_{j}(\bar{z})-a_{m}}} .
$$


The infinite product (3-3) converges uniformly in every compact subset of $\bar{D}$; see [Mityushev and Rogosin 2000, p. 153]. The multipliers in the infinite product (3-3) are ordered according to increasing level.

Consider a fixed $s$ from the set $\{1,2, \ldots, n\}$. The harmonic measure $\alpha_{s}(\zeta)$ associated to $D$ satisfies the boundary conditions

$$
\alpha_{s}(z)=\delta_{s k}, \quad\left|z-a_{k}\right|=r_{k}, k=1,2, \ldots, n,
$$

where $\delta_{s k}$ is the Kronecker symbol. $\alpha_{s}(z)$ is explicitly written in [Mityushev and Rogosin 2000, p. 153, Theorem 4.10] as follows:

$$
\alpha_{s}(z)=\sum_{m=1}^{n} A_{s m} \operatorname{Re}\left[\psi_{m}(z)+\ln \left(z-a_{m}\right)\right]+A_{s},
$$

where $\psi_{m}(z)$ has the form (3-3), and the real constants $A_{s m}$ and $A_{s}$ are described in the theorem just cited.

Following Chapter 4 of [Mityushev and Rogosin 2000] we briefly describe how to deduce (3-7). The harmonic measure $\alpha_{s}(z)$ as a harmonic function in $D$ can be represented in the form

$$
\alpha_{s}(z)=\operatorname{Re} \varphi(z)+\sum_{m=1}^{n} A_{s m} \ln \left|z-a_{m}\right|+A_{s},
$$

where $\varphi(z)$ is analytic in $D$ and continuously differentiable in its closure $(\varphi \in$ $\left.A^{1}(D)\right)$ and

$$
\varphi\left(z_{0}\right)=0 .
$$

$A_{s m}$ are real constants having zero sum. The real constant $A_{s}$ is introduced for convenience. It can be included into $\operatorname{Re} \varphi(z)$. Substitution of (3-8) into (3-6) yields the boundary value problem

(3-10) $\operatorname{Re} \varphi(z)+\sum_{m=1}^{n} A_{s m} \ln \left|z-a_{m}\right|+A_{s}=\delta_{s k}, \quad\left|z-a_{k}\right|=r_{k}, k=1,2, \ldots, n$.

It can be reduced, first, to the $\mathbb{R}$-linear conjugation problem

$$
\varphi(z)=\varphi_{k}(z)-\overline{\varphi_{k}(z)}+f(z), \quad\left|z-a_{k}\right|=r_{k}, k=1,2, \ldots, n,
$$

where $\varphi_{k} \in A^{1}\left(D_{k}\right), f(z)=\delta_{s k}-A_{s}-A_{s k} \ln r_{k}-\sum_{m \neq k} A_{s m} \ln \left(z-a_{m}\right), z \in D_{k}$.

The problem (3-10) is equivalent to the problem (3-11), i.e., the problem (3-10) is solvable if and only if (3-11) is solvable. If $\varphi(z)$ is a solution of (3-10), it is also a solution of (3-11) in $D$, and a solution of (3-11) in $D_{k}$ can be found from 
the following simple problem for the disc $D_{k}$ with respect to function $2 \operatorname{Im} \varphi_{k}(z)$ harmonic in $D_{k}$

$$
2 \operatorname{Im} \varphi_{k}(z)=\operatorname{Im}(\varphi(z)-f(z)), \quad\left|z-a_{k}\right|=r_{k} .
$$

The problem (3-12) has a unique solution up to an arbitrary additive real constant.

We introduce the following function:

$$
\Phi(z):=\left\{\begin{array}{l}
\varphi_{k}(z)+\sum_{m \neq k}\left(\overline{\varphi_{m}\left(z_{(m)}^{*}\right)}-\overline{\varphi_{m}\left(\left(z_{0}\right)_{(m)}^{*}\right)}\right)-\overline{\varphi_{k}\left(\left(z_{0}\right)_{(k)}^{*}\right)}+f(z), \\
\varphi(z)+\sum_{m=1}^{n}\left(\overline{\varphi_{m}\left(z_{(m)}^{*}\right)}-\overline{\varphi_{m}\left(\left(z_{0}\right)_{(m)}^{*}\right)}\right), \quad z \in D .
\end{array}\right.
$$

The jump across the circle $\left|t-a_{k}\right|=r_{k}$ can be calculated as

$$
\Delta_{k}:=\Phi^{+}(t)-\Phi^{-}(t),
$$

where $\Phi^{+}(t):=\lim _{z \rightarrow t} z \in D \Phi(z)$ and $\Phi^{-}(t):=\lim _{z \rightarrow t} z \in D_{k} \Phi(z)$. Using (3-11) we get $\Delta_{k}=0$. It follows from the analytic continuation principle that $\Phi(z)$ is analytic in the extended complex plane. Then Liouville's theorem implies that $\Phi(z)$ is a constant. Using (3-9) we calculate $\Phi\left(z_{0}\right)=0$, hence $\Phi(z) \equiv 0$. The definition of $\Phi(z) \equiv 0$ in $\left|z-a_{k}\right| \leq r_{k}$ yields the following system of functional equations:

$$
\begin{aligned}
\varphi_{k}(z)=-\sum_{m \neq k}\left(\overline{\varphi\left(z_{(m)}^{*}\right)}-\overline{\varphi\left(\left(z_{0}\right)_{(m)}^{*}\right)}\right)+g(z), \\
\quad\left|z-a_{k}\right| \leq r_{k}, \quad k=1,2, \ldots, n,
\end{aligned}
$$

where $g(z)=-f(z)+\overline{\varphi\left(\left(z_{0}\right)_{(k)}^{*}\right)}$. Equations (3-13) $\mathbb{R}$-linearly relate the values of $\varphi_{k}$ at $z \in \bar{D}_{k}$ with the values of $\varphi_{m}(m \neq k)$ at the symmetric points $z_{(m)}^{*} \in D_{m}$. If $\varphi_{k}(z)$ is known, $\varphi(z)$ has the form

$$
\varphi(z)=-\sum_{m \neq k}\left(\overline{\varphi\left(z_{(m)}^{*}\right)}-\overline{\varphi\left(\left(z_{0}\right)_{(m)}^{*}\right)}\right), \quad z \in D .
$$

Lemma 3.1 [Mityushev and Rogosin 2000, p. 147]. The system of functional equations (3-13) for any $g \in A^{1}\left(\bigcup_{m=1}^{n} \bar{D}_{m}\right)$ has a unique solution. This solution can be found by the method of successive approximations. The approximations converge uniformly in $\bigcup_{m=1}^{n} \bar{D}_{m}$.

The straightforward application of successive approximations to (3-13), combined with the use of (3-14), yield (3-7) with (3-3)-(3-5).

Green's function. Consider the function $\omega(z, \zeta)$ defined by the formulas (4.4.27) or (4.4.28) from [Mityushev and Rogosin 2000, p. 156]

$$
\omega(z, \zeta)=\ln \prod_{j=1}^{\infty} \omega_{j}(z, \zeta)
$$


where

$$
\omega_{j}(z, \zeta)=\left\{\begin{array}{l}
\frac{\zeta-\gamma_{j}(z)}{\zeta-\gamma_{j}\left(z_{0}\right)}, \text { if level of } \gamma_{j} \text { is even } \\
\frac{\zeta-\gamma_{j}\left(\bar{z}_{0}\right)}{\overline{\zeta-\gamma_{j}(\bar{z})}}, \text { if level of } \gamma_{j} \text { is odd. }
\end{array}\right.
$$

The infinite product (3-15) converges uniformly on $z$ in $\bar{D}$ for each fixed $\zeta \in D$ (uniformly on each compact subset of $\bar{D}-\{\zeta\}$ if $\zeta \in \partial D$ ). Let $\mathscr{G} \subset \mathscr{S}$ be the subgroup consisting of $\gamma_{j}$ of the even level, $\mathscr{F} \subset \mathscr{Y}$ be the set of $\gamma_{j}$ of the odd level.

Lemma 3.2 [Mityushev and Rogosin 2000, p. 154]. The complex Green's function $M(z, \zeta)$ associated with $D$ is represented in the form

$$
M(z, \zeta)=\sum_{k=1}^{n} \alpha_{k}(\zeta)\left[\psi_{k}(z)+\ln \left(z-a_{k}\right)\right]-\omega(z, \zeta)-\ln (z-\zeta)+A(\zeta)
$$

The proof of this lemma is based on the method of functional equations described in the previous subsection.

Let $G(z, \zeta)$ be the real Green's function. Then

$$
G(z, \zeta)=\frac{1}{2}[M(z, \zeta)+\overline{M(z, \zeta)}]
$$

We want to calculate $\frac{\partial^{2} G}{\partial z \partial \bar{\zeta}}$. The function $M(z, \zeta)$ is analytic on $z$, i.e.,

$$
\frac{\partial \bar{M}}{\partial z}=0 .
$$

It follows from (3-18) and (3-19) that

$$
\frac{\partial^{2} G}{\partial z \partial \bar{\zeta}}=\frac{1}{2} \frac{\partial^{2} M}{\partial z \partial \bar{\zeta}}
$$

By using (3-17), we obtain

$$
\frac{\partial^{2} M}{\partial z \partial \bar{\zeta}}=\sum_{k=1}^{n} \frac{\partial \alpha_{k}(\zeta)}{\partial \bar{\zeta}}\left(\psi_{k}^{\prime}(z)+\frac{1}{z-a_{k}}\right)-\frac{\partial^{2} \omega(z, \zeta)}{\partial z \partial \bar{\zeta}} .
$$

Here we use the evident equalities

$$
\frac{\partial \ln (z-\zeta)}{\partial \bar{\zeta}}=0, \quad \frac{\partial A(\zeta)}{\partial z}=0 .
$$

Differentiating (3-7) we obtain

$$
\frac{\partial \alpha_{k}}{\partial \bar{\zeta}}=\frac{1}{2} \sum_{m=1}^{n} A_{m}\left(\overline{\psi_{m}^{\prime}(\zeta)}+\frac{1}{\overline{\zeta-a_{m}}}\right)
$$


where the prime means the derivative on $z$ or on $\zeta$ (but not on complex conjugated variables). Then (3-21) becomes

$$
\frac{\partial^{2} M}{\partial z \partial \bar{\zeta}}=\sum_{k=1}^{n} \sum_{m=1}^{n} A_{m}\left(\overline{\psi_{m}^{\prime}(\zeta)}+\frac{1}{\overline{\zeta-a_{m}}}\right)\left(\psi_{k}^{\prime}(z)+\frac{1}{z-a_{k}}\right)-\frac{\partial^{2} \omega(z, \zeta)}{\partial z \partial \bar{\zeta}}
$$

We calculate $\frac{\partial^{2} \omega(z, \zeta)}{\partial z \partial \bar{\zeta}}$ using the formulas (3-15) and (3-16)

$$
\frac{\partial^{2} \omega(z, \zeta)}{\partial z \partial \bar{\zeta}}=-\sum_{\gamma_{j} \in \mathscr{F}} \frac{\bar{\gamma}_{j}^{\prime}(z)}{\left(\bar{\zeta}-\bar{\gamma}_{j}(z)\right)^{2}}
$$

We now proceed to calculate $\psi_{m}^{\prime}(z)$, where $\psi_{m}(z)$ has the form (3-3). Differentiate the logarithms of (3-4)-(3-5)

$$
\Psi_{m}^{(j)}(z):=\left(\ln \psi_{m}^{(j)}\right)^{\prime}(z)=\left\{\begin{array}{cl}
\frac{\gamma_{j}^{\prime}(z)}{\gamma_{j}(z)-a_{m}} & \text { for } \gamma_{j} \in \mathscr{G}, \\
-\frac{\bar{\gamma}_{j}^{\prime}(z)}{\bar{\gamma}_{j}(z)-a_{m}} & \text { for } \gamma_{j} \in \mathscr{F} .
\end{array}\right.
$$

Then (3-20) and (3-24) yield

$$
\text { (3-27) } \frac{\partial^{2} G}{\partial z \partial \bar{\zeta}}=\frac{1}{2} \sum_{k=1}^{n} \sum_{m=1}^{n} A_{m} \overline{\sum_{\gamma_{j} \in \mathscr{T}_{m}}^{\infty} \Psi_{m}^{(j)}(\zeta)} \sum_{\gamma_{j} \in \mathscr{S}_{m}} \Psi_{m}^{(j)}(z)+\frac{1}{2} \sum_{\gamma_{j} \in \mathscr{F}} \frac{\bar{\gamma}_{j}^{\prime}(z)}{\left(\bar{\zeta}-\bar{\gamma}_{j}(z)\right)^{2}} \text {, }
$$

where $\Psi_{m}^{(j)}(z)$ has the form (3-26).

Bergman kernel. Using (2-11) and (3-27) we obtain the following result.

Theorem 3.3. The Bergman kernel $K(z, w)$ associated with $D$ is given by (3-28)

$$
K(z, \zeta)=-\frac{1}{\pi} \sum_{k=1}^{n} \sum_{m=1}^{n} A_{m} \overline{\sum_{\gamma_{j} \in \mathscr{S}_{m}}^{\infty} \Psi_{m}^{(j)}(\zeta)} \sum_{\gamma_{j} \in \mathscr{S}_{m}} \Psi_{m}^{(j)}(z)-\frac{1}{\pi} \sum_{\gamma_{j} \in \mathscr{F}} \frac{\bar{\gamma}_{j}^{\prime}(z)}{\left(\bar{\zeta}-\bar{\gamma}_{j}(z)\right)^{2}},
$$

where $\Psi_{m}^{(j)}(z)$ has the form (3-26).

Example 3.4. Consider the simply connected domain $D=\{z \in \overline{\mathbb{C}}:|z|>1\}$. Then we have the two-element group of inversions $\gamma_{0}(z)=z, \gamma_{1}(\bar{z})=1 / \bar{z}$. The constant $A_{1}$ is equal to zero (see (4.4.3) of [Mityushev and Rogosin 2000, p. 149]) and (3-28) becomes

$$
K(z, \zeta)=-\frac{1}{\pi} \frac{\bar{\gamma}_{1}^{\prime}(z)}{\left(\bar{\zeta}-\bar{\gamma}_{1}(z)\right)^{2}}=\frac{1}{\pi} \frac{1}{(1-z \bar{\zeta})^{2}}
$$


We are not aware that any short demonstration exists which shows that Theorem 3.3 yields the well-known formula (1-4) for doubly connected domains under appropriate conformal mappings.

Bell [1995] showed that the Bergman kernel is rational if and only if the associated domain is simply connected and there is a rational biholomorphic mapping of the given domain onto the unit disc. He proved [1999] that when the given domain is a finitely connected planar domain such that no boundary component is a point, the corresponding Bergman kernel is algebraic if and only if there is a proper holomorphic mapping of the given domain onto the unit disc which is algebraic.

Thus, for a circular domain $D$ defined by (2-1)-(2-2), if $n=1, D$ is biholomorphic to the unit disc with a rational biholomorphic mapping $f(z)=1 /\left(z-a_{1}\right)$, and hence the Bergman kernel is rational; see Example 3.4 above. We know that every unbounded $n$-connected domain $D$ is biholomorphic to a bounded $n$-connected domain. Hence, if $n>1$, the Bergman kernel for $D$ is not rational, but is algebraic if there is an algebraic proper holomorphic mapping from $D$ onto the unit disc.

\section{Acknowledgments}

The authors are grateful to the referees and Guy Roos for remarks improving the presentation of the results.

\section{References}

[Aharonov et al. 2005] D. Aharonov, C. Bénéteau, D. Khavinson, and H. Shapiro, "Extremal problems for nonvanishing functions in Bergman spaces", pp. 59-86 in Selected topics in complex analysis, edited by V. Eiderman and M. Samokhin, Oper. Theory Adv. Appl. 158, Birkhäuser, Basel, 2005. MR 2006i:30047 Zbl 1089.30032

[Ball and Clancey 1996] J. A. Ball and K. F. Clancey, "Reproducing kernels for Hardy spaces on multiply connected domains", Integral Eq. Operator Theory 25:1 (1996), 35-57. MR 97f:46042 Zbl 0867.30038

[Bell 1992] S. R. Bell, The Cauchy transform, potential theory, and conformal mapping, Studies in Advanced Mathematics, CRC Press, Boca Raton, FL, 1992. MR 94k:30013 Zbl 0765.30026

[Bell 1995] S. R. Bell, "Complexity of the classical kernel functions of potential theory", Indiana Univ. Math. J. 44:4 (1995), 1337-1369. MR 97g:30009 Zbl 0862.31001

[Bell 1999] S. R. Bell, "Finitely generated function fields and complexity in potential theory in the plane", Duke Math. J. 98:1 (1999), 187-207. MR 2000i:30015 Zbl 0948.30015

[Bergman 1950] S. Bergman, The kernel function and conformal mapping, Mathematical Surveys 5, Amer. Math. Soc., New York, 1950. Reprinted 1970. MR 12,402a Zbl 0040.19001

[Boas et al. 1999] H. P. Boas, S. Fu, and E. J. Straube, “The Bergman kernel function: explicit formulas and zeroes”, Proc. Amer. Math. Soc. 127:3 (1999), 805-811. MR 99f:32037 Zbl 0919.32013

[Conway 1978] J. B. Conway, Functions of one complex variable, 2nd ed., Graduate Texts in Mathematics 11, Springer, New York, 1978. MR 80c:30003 Zbl 0277.30001 
[D’Angelo 1994] J. P. D'Angelo, “An explicit computation of the Bergman kernel function”, $J$. Geom. Anal. 4:1 (1994), 23-34. MR 95a:32039 Zbl 0794.32021

[Epstein 1965] B. Epstein, Orthogonal families of analytic functions, Macmillan, New York, 1965. MR 31 \#4916 Zbl 0145.08901

[Greene and Krantz 2002] R. E. Greene and S. G. Krantz, Function theory of one complex variable, 2nd ed., Graduate Studies in Mathematics 40, Amer. Math. Soc., Providence, RI, 2002. MR 2003e:30001 Zbl 0988.30001

[Hua 1963] L.-K. Hua, Harmonic analysis of functions of several complex variables in classical domains, Translations of Math. Monographs 6, Amer. Math. Soc., Providence, 1963. MR 23 \#A3277 Zbl 0112.07402

[Mityushev and Rogosin 2000] V. V. Mityushev and S. V. Rogosin, Constructive methods for linear and nonlinear boundary value problems for analytic functions: Theory and applications, Monographs and Surveys in Pure and Applied Mathematics 108, Chapman \& Hall/CRC, Boca Raton, FL, 2000. MR 2001d:30075 Zbl 0957.30002

[Oeljeklaus et al. 1997] K. Oeljeklaus, P. Pflug, and E. H. Youssfi, "The Bergman kernel of the minimal ball and applications”, Ann. Inst. Fourier (Grenoble) 47:3 (1997), 915-928. MR 98d:32028 Zbl 0873.32025

[Schiffer 1946] M. Schiffer, "The kernel function of an orthonormal system", Duke Math. J. 13 (1946), 529-540. MR 8,371a Zbl 0060.23708

[Shabat 1985] B. V. Shabat, Введение в комплексный анализ, 1: функции одного переменного, 3rd ed., Nauka, Moscow, 1985. MR 88b:32001 Zbl 0574.30001

Received August 3, 2006. Revised May 22, 2007.

\author{
MOONJA JEONG \\ Department of Mathematics \\ THE UNIVERSITY OF SUWON \\ SUWON P.O.BOX 77 \\ KYUNGKIDO 440-600 \\ KOREA \\ mjeong@suwon.ac.kr \\ VLADIMIR MITYUSHEV \\ DEPARTMENT OF MATHEMATICS \\ Pedagogical ACADEMy \\ UL. PODCHORAZYCH 2 \\ KRAKÓW 30-084 \\ POLAND \\ vmityu@yahoo.com
}

